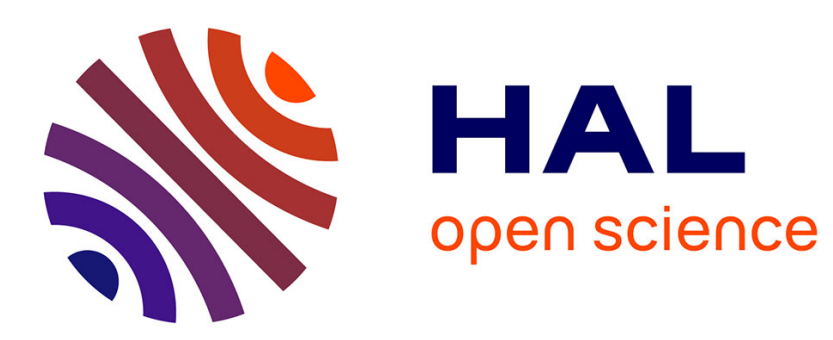

\title{
A new formulation for solving 3-D time dependent rolling contact problems of a rigid body on a viscoelastic half-space
}

Hai Ping Yin, Julien Cesbron, Q H Bui

\section{- To cite this version:}

Hai Ping Yin, Julien Cesbron, Q H Bui. A new formulation for solving 3-D time dependent rolling contact problems of a rigid body on a viscoelastic half-space. Mechanics Research Communications, 2015, 64, pp.8-14. 10.1016/j.mechrescom.2014.12.006 . hal-01470386

\section{HAL Id: hal-01470386 \\ https://hal.science/hal-01470386}

Submitted on 17 Feb 2017

HAL is a multi-disciplinary open access archive for the deposit and dissemination of scientific research documents, whether they are published or not. The documents may come from teaching and research institutions in France or abroad, or from public or private research centers.
L'archive ouverte pluridisciplinaire HAL, est destinée au dépôt et à la diffusion de documents scientifiques de niveau recherche, publiés ou non, émanant des établissements d'enseignement et de recherche français ou étrangers, des laboratoires publics ou privés. 


\section{Accepted Manuscript}

Title: A new formulation for solving 3-D time dependent rollingcontact problems of a rigid body on a viscoelastic half-space

Author: H.P. Yin J. Cesbron Q.H. Bui

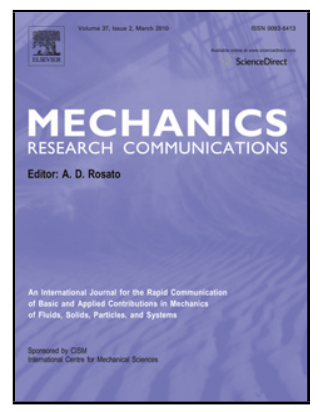

PII: S0093-6413(14)00156-6

DOI: http://dx.doi.org/doi:10.1016/j.mechrescom.2014.12.006

Reference: MRC 2921

To appear in:

Received date: $\quad 9-1-2013$

Revised date: 6-11-2014

Accepted date: $\quad$ 28-12-2014

Please cite this article as: H.P. Yin, J. Cesbron, Q.H. Bui, A new formulation for solving 3-D time dependent rollingcontact problems of a rigid body on a viscoelastic half-space, Mechanics Research Communications (2015), http://dx.doi.org/10.1016/j.mechrescom.2014.12.006

This is a PDF file of an unedited manuscript that has been accepted for publication. As a service to our customers we are providing this early version of the manuscript. The manuscript will undergo copyediting, typesetting, and review of the resulting proof before it is published in its final form. Please note that during the production process errors may be discovered which could affect the content, and all legal disclaimers that apply to the journal pertain. 
- We propose a new formulation for the steady viscoelastic contact problem in rolling conditions without friction.

- The numerical method is efficient for rollers of arbitrary shape.

- The method is also valid for unstationnary rolling conditions. 


\title{
A new formulation for solving 3-D time dependent rolling contact problems of a rigid body on a viscoelastic half-space
}

\author{
H.P. Yin ${ }^{\mathrm{b}}$, J. Cesbron ${ }^{\mathrm{a}, *}$, Q.H. Bui ${ }^{\mathrm{b}}$ \\ ${ }^{a}$ LUNAM Université, IFSTTAR, AME, LAE, F-44344 Bouguenais, France \\ ${ }^{b}$ Université Paris-Est, Laboratoire Navier (UMR 8205), CNRS, ENPC, IFSTTAR, F-77455 Marne-la-Vallée,
} France

\begin{abstract}
The paper deals with a new formulation for solving the rolling contact problem without friction of a rigid body on a viscoelastic half-space in three dimensions. Assuming that the material behavior is independent of time for a sufficiently short time duration, the viscoelastic contact problem is transformed into elastic like problems. Then the contact problem is solved using a direct numerical method at each time step. The convergence of the method in time and space is good for a spherical indenter. The dissymmetry of the contact patch due to hysteresis was found in three dimensions for the spherical indenter and two cylinders of different width. Finally the method was tested for a sinusoidal varying speed and shows a good efficiency.
\end{abstract}

Keywords: Rolling contact, Viscoelasticity, Numerical simulations

\section{Introduction}

Rolling systems such as car tyres and conveyer systems widely use elastomers. Therefore the time dependent behavior of elastomers should be taken into account when computing rolling contact for such systems. The viscoelastic rolling contact is also a fundamental problem which was first investigated by the experimental work of Tabor (1952) and the studies of Hunter (1961) and Morland (1962) on the rolling contact of a rigid cylinder on a viscoelastic half-space. However most of these studies are restricted to cylinder cases for two dimensional problems. This paper presents a new algorithm for computing the 3-D time dependent rolling contact between a rigid body and a viscoelastic half-space. The friction is not taken into account in this work.

Since the middle of the 80's, Finite Element Methods (FEM) have been used to solve viscoelastic rolling contact problems. Padovan and Paramadilok (1985) developed a travelling finite element strategy based on moving total Lagrangian coordinates to handle transient and steady viscoelastic rolling contact. Oden and Lin (1986) studied the contact of hyperelastic and viscoelastic rolling cylinders on a rough foundation. The Arbitrary Lagrangian Eulerian (ALE) formulation and a finite element approach were applied to rolling contact problems by Nackenhorst (2004) and later applied to rolling noise simulations (Brinkmeier et al., 2008).

On the other hand, Boundary Element Methods (BEM) were used by Kalker to solve the elastic rolling contact problems (Kalker, 1990) and later the problem of rolling viscoelastic

*Corresponding author: Tel: +3324084 5662

Email address: julien.cesbron@ifsttar.fr (J. Cesbron) 
multilayered cylinders (Kalker, 1991). The contact stresses between viscoelastic cylinders were also computed with BEM by Wang (1993) and González and Abascal (2006).

Though many formulations and strategies have been developed to solve viscoelastic rolling problems, most of them are restricted to 2-D and/or stationary rolling. In this paper, a new formulation is proposed for the 3-D time dependent rolling contact between a rigid body and a viscoelastic half-space. The friction will be neglected in the present work and only the normal pressure is studied. Based on the assumption that the material behavior is independent of time for a sufficiently short time duration, the viscoelastic contact problem is transformed into elastic like problems. As a result, numerical methods developed for solving elastic contact problems can be used.

In section 2, the formulation of viscoelastic contact is described without the rolling conditions for facilitating the comprehension. Then the rolling conditions are introduced in a Lagrangian coordinates system. Section 3 presents the numerical algorithm that uses the principle of direct matrix inversion methods (Johnson, 1985). Numerical results are given in Section 4 for a rolling sphere and rolling cylinders of different lengths. The method and the examples are presented in terms of a displacement-control problem. Transient rolling and sinusoidal speed rolling are considered to show the efficiency of the formulation.

\section{Formulation of the viscoelastic rolling contact problem}

The problem of the contact between a rigid solid and a viscoelastic half-space is considered in frictionless conditions. The present normal displacement $w$ at any point $(x, y)$ within the contact area depends on the pressure distribution history $p(\xi, \eta, \tau)$ :

$$
w(x, y, t)=\int_{0}^{t} J(t-\tau) \frac{d}{d \tau}\left[\iint_{S_{m}} T(x, y ; \xi, \eta) p(\xi, \eta, \tau) d s\right] d \tau
$$

where $S_{m}$ is the maximal contact area, $J$ is the creep function and $T(x, y ; \xi, \eta)$ is the influence function which designates the displacement induced at point $(x, y)$ by a unit point force at $(\xi, \eta)$.

The influence function is then given by the Boussinesq's fundamental solution:

$$
T(x, y ; \xi, \eta)=\frac{(1-\nu)}{\pi \sqrt{(x-\xi)^{2}+(y-\eta)^{2}}}
$$

We suppose that the pressure distribution up to $t-\Delta t$ is known, and propose to compute the pressure distribution at instant $t$. For the sake of simplicity, we use the following notation for any two instants $t_{1}$ and $t_{2}$ :

$$
I_{t_{1}}^{t_{2}}=\int_{t_{1}}^{t_{2}} J(t-\tau) \frac{d}{d \tau}\left[\iint_{S_{m}} T(x, y ; \xi, \eta) p(\xi, \eta, \tau) d s\right] d \tau
$$

Then the following equation holds:

$$
I_{0}^{t}=I_{0}^{t-\Delta t}+I_{t-\Delta t}^{t}
$$

In the latter equation, the first term $I_{0}^{t-\Delta t}$ can be computed from the pressure distribution history before $t-\Delta t$. The second term $I_{t-\Delta t}^{t}$ will be studied. When $\tau$ varies from $t-\Delta t$ to $t$, 
$t-\tau$ varies from $\Delta t$ to 0 . We assume that if the time duration $\Delta t$ is sufficiently short, the creep function is constant:

$$
J(t-\tau) \approx J(0) \quad \text { for } \quad t-\Delta t<\tau<t
$$

Then one can derive:

$$
I_{t-\Delta t}^{t} \approx J(0) \int_{S(t)} T(x, y ; \xi, \eta) p(\xi, \eta, t) d s-J(0) \int_{S_{m}} T(x, y ; \xi, \eta) p(\xi, \eta, t-\Delta t) d s
$$

where $S(t)$ denotes the present contact area.

By introducing the preceding displacement $u(x, y, t)$ which represents the contribution to $w(x, y, t)$ due to the pressure distribution history between 0 and $t-\Delta t$ :

$$
u(x, y, t)=I_{0}^{t-\Delta t}-J(0) \int_{S_{m}} T(x, y ; \xi, \eta) p(\xi, \eta, t-\Delta t) d s
$$

and taking into account the above equations, Eq. (1) becomes:

$$
w(x, y, t) \approx u(x, y, t)+J(0) \int_{S(t)} T(x, y ; \xi, \eta) p(\xi, \eta, t) d s
$$

Then the present pressure distribution can be determined by solving the unilateral contact problem given by the complementary relations between the gap and the normal pressure:

$$
\left\{\begin{array}{l}
\forall(x, y) \in S(t), \delta(t)-z(x, y)-w(x, y, t)=0 \text { and } p(x, y, t)>0 \\
\forall(x, y) \in \bar{S}(t), \delta(t)-z(x, y)-w(x, y, t)>0 \text { and } p(x, y, t)=0
\end{array}\right.
$$

where $\delta(t)$ is the normal penetration of the indenter in the half-space (i.e. the normal displacement at the tip of the indenter), $z(x, y)$ described the geometry of the surface of the indenter, $w(x, y, t)$ is the present normal displacement as defined in Eq. (1) and $\bar{S}(t)$ is the surface of the half-space where there is no contact. Then the contact condition on the gap in Eq. (9) combined with Eq. (8) leads to:

$$
\forall(x, y) \in S(t), J(0) \int_{S(t)} T(x, y ; \xi, \eta) p(\xi, \eta, t) d s \approx \delta(t)-z(x, y)-u(x, y, t)
$$

Since $u(x, y, t)$ is assumed to be known, this problem can be seen as an elastic like contact problem at instant $t$.

In rolling conditions, one can follow a point in the contact plane by introducing the coordinates below:

$$
X(x, y, t)=x+\int_{0}^{t} V_{x}(\tau) d \tau, \quad Y(x, y, t)=y+\int_{0}^{t} V_{y}(\tau) d \tau
$$

where $V_{x}$ and $V_{y}$ designate the speed of the rolling solid in the contact plan. The frames of coordinates are illustrated in Fig. 1.

Then in rolling conditions one should replace in all equations $(x, y)$ with $(X, Y)$ and $z(x, y)$ with $Z(X, Y, t)$ which describes the surface profile of the rolling solid potentially in contact with the half-space at the present instant. For instance Eq. (1) becomes:

$$
w(X, Y, t)=\int_{0}^{t} J(t-\tau) \frac{d}{d \tau}\left[\iint_{S_{m}} T(X, Y ; \xi, \eta) p(\xi, \eta, \tau) d s\right] d \tau
$$




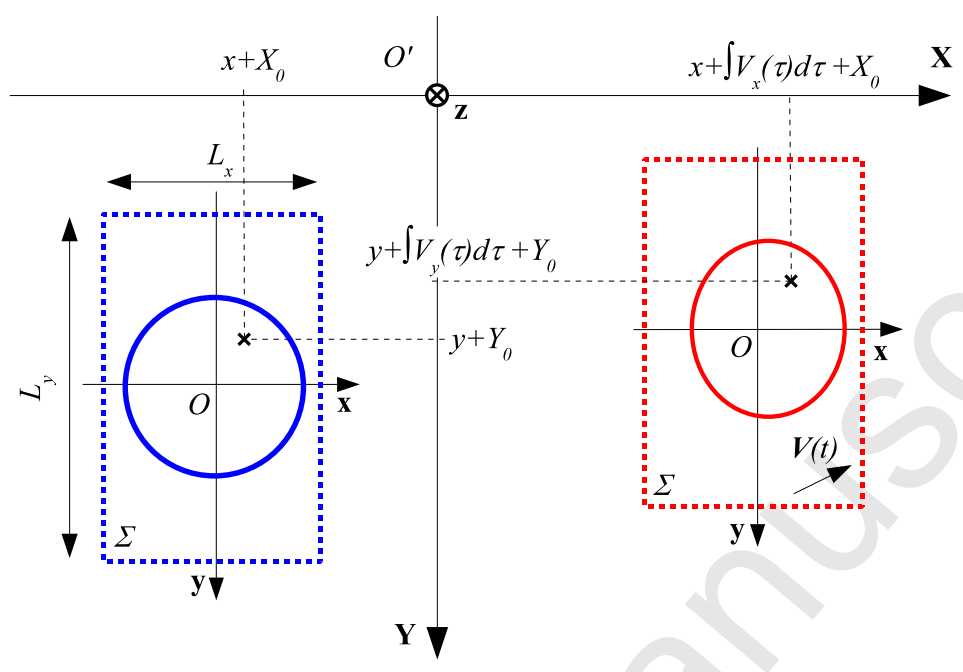

Fig. 1: Frames of coordinates for the rolling problem.

and the contact problem becomes:

$$
\forall(X, Y) \in S(t),\left\{\begin{array}{l}
J(0) \int_{S(t)} T(X, Y ; \xi, \eta) p(\xi, \eta, t) d s \approx \delta(t)-Z(X, Y, t)-u(X, Y, t) \\
p(X, Y, t)>0
\end{array}\right.
$$

Like Eq. (10), Eq. (13) is an elastic like problem which can be solved by using classical methods such as the Matrix Inversion Method (Johnson, 1985; Kalker, 1990).

\section{Numerical procedure}

The surface involving the potential contact area of size $L_{x} \times L_{y}$ was meshed using $n=n_{x} n_{y}$ rectangular elements of dimensions $d x=L_{x} / n_{x}$ and $d y=L_{y} / n_{y}$ centered at $\left(x_{i}, y_{i}\right)$ and with uniform pressure on each element.

The influence coefficient $T_{i j}$ expresses the displacement at a point $\left(x_{i}, y_{i}\right)$ due to an unit uniform pressure on the element centered at point $\left(x_{j}, y_{j}\right)$. For a half-space, $T_{i j}$ can be calculated using Love's results Love (1952):

$$
T_{i j}=\frac{(1-\nu)}{\pi} \int_{y_{j}-\frac{d y}{2}}^{y_{j}+\frac{d y}{2}} \int_{x_{j}-\frac{d x}{2}}^{x_{j}+\frac{d x}{2}} \frac{d \xi d \eta}{\sqrt{\left(x_{i}-\xi\right)^{2}+\left(y_{i}-\eta\right)^{2}}}
$$

The time discretization is $\tau=k d \tau$. Then equation (9) is written in the form:

$$
\begin{aligned}
& J(0) \sum_{j=1}^{n} T_{i j} p_{j}^{l}=\delta^{l}-z_{i}-v_{i}^{l-1}, \quad i=1 \ldots n \\
& v_{i}^{l-1}=\sum_{k=1}^{l-1} J((l-k) d \tau) \sum_{j=1}^{n} T_{i j}\left[p_{j}^{k}-p_{j}^{k-1}\right]-J(0) \sum_{j=1}^{n} T_{i j} p_{j}^{l-1}
\end{aligned}
$$


where $p_{j}^{k}=p\left(x_{j}, y_{j}, k d \tau\right), \delta^{l}=\delta(l d \tau), z_{i}=z\left(x_{i}, y_{i}\right)$. These equations are the same as in Kozhevnikov et al. (2008).

In rolling conditions, Eq. (11) becomes:

$$
X_{i}^{k}=x+\sum_{m=1}^{k} V_{x}(m d \tau) d \tau, \quad Y_{i}^{k}=y+\sum_{m=1}^{k} V_{y}(m d \tau) d \tau
$$

and the influence function becomes:

$$
T_{i j}^{k}=\frac{(1-\nu)}{\pi} \int_{Y_{j}^{k}-\frac{d y}{2}}^{Y_{j}^{k}+\frac{d y}{2}} \int_{X_{j}^{k}-\frac{d x}{2}}^{X_{j}^{k}+\frac{d x}{2}} \frac{d \xi d \eta}{\sqrt{\left(X_{i}^{l}-\xi\right)^{2}+\left(Y_{i}^{l}-\eta\right)^{2}}}
$$

The system of equations to be solved is:

$$
\begin{aligned}
& J(0) \sum_{j=1}^{n} T_{i j}^{l} p_{j}^{l}=\delta^{l}-Z_{i}-v_{i}^{l-1}, \quad i=1 \ldots n \\
& v_{i}^{l-1}=\sum_{k=1}^{l-1} J((l-k) d \tau) \sum_{j=1}^{n}\left[T_{i j}^{k} p_{j}^{k}-T_{i j}^{k-1} p_{j}^{k-1}\right]-J(0) \sum_{j=1}^{n} T_{i j}^{l-1} p_{j}^{l-1}
\end{aligned}
$$

The contact problem is solved using the classical Matrix Inversion Method (Johnson, 1985; Kalker, 1990), as described in Kozhevnikov et al. (2008).

\section{Results}

This section presents first the results of the numerical method in terms of convergence. Then the results of the method are compared with the simple viscoelastic foundation model. The influence of the geometry of the indenter and rolling at a varying speed are also studied. The calculations were performed in the time interval $t \in[0, T]$. The viscoelasticity of the material was described using the standard linear solid, i.e. the Kelvin-Voigt model with delayed elasticity. This behavior is represented in Fig. 2 by a spring of modulus $E_{1}$ in series with a spring of modulus $E_{2}$ in parallel with a dashpot of viscosity $\eta$. Then the relaxation and creep functions,

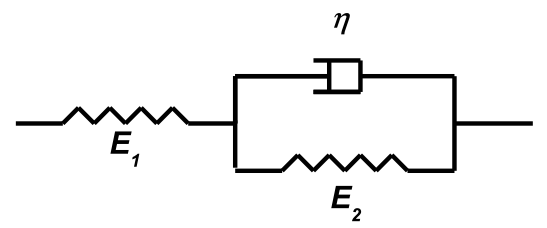

Fig. 2: Standard linear viscoelastic solid used in the study.

noted $G(t)$ and $J(t)$ respectively, can be written:

$$
\left\{\begin{aligned}
G(t) & =E_{\infty}+\left(E_{0}-E_{\infty}\right) e^{-\frac{t}{\tau_{c}}} \\
J(t) & =\frac{1}{E_{\infty}}-\frac{E_{0}-E_{\infty}}{E_{0} E_{\infty}} e^{-\frac{E_{\infty} t}{E_{0} \tau_{c}}}
\end{aligned}\right.
$$


where $\tau_{c}=\eta / E_{2}$ is the characteristic relaxation time, $E_{0}=E_{1}$ is the instantaneous Young's modulus and $E_{\infty}=E_{1} E_{2} /\left(E_{1}+E_{2}\right)$ is the Young's modulus in static conditions after relaxation. For the calculation, the values of $E_{0}$ and $E_{\infty}$ were such that $E_{0}=2 E_{\infty}=15 \mathrm{MPa}$. The characteristic time was $\tau_{c}=0.001 \mathrm{~s}$ for a duration of rolling $T=0.1 \mathrm{~s}$. The rolling was considered on a straight line trajectory along the $(O, X)$ axis, which means that $V_{y}=0 \mathrm{~m} / \mathrm{s}$ for the calculation. The contact problem was solved with a constant value of the penetration $\delta$.

\subsection{Convergence of the algorithm}

The convergence of the algorithm was studied using a rigid spherical indenter of radius $R=150$ $\mathrm{mm}$ rolling at a constant speed $V_{x}$ on the viscoelastic half-space. The penetration $\delta$ was $4.2 \mathrm{~mm}$, i.e. $\delta / R=0.028$, which guarantees small deformations. The convergence in the space domain $(x, y)$ was studied in Kozhevnikov et al. (2008) and leads to the discretization rule $d x / a \leq 0.1$, where $a$ is the radius of the contact area in the elastic case. Thus, since $a$ is around $30 \mathrm{~mm}, d x$ and $d y$ were fixed with a maximum value of $3.0 \mathrm{~mm}$.

The convergence in the time domain was first tested for $V_{x}=15 \mathrm{~m} / \mathrm{s}(54 \mathrm{~km} / \mathrm{h})$, with a fine spatial resolution $d x=d y=1.5 \mathrm{~mm}$. The number of segments $n_{t}$ in the time interval $[0, T]$ varied between 11 and 301 . The total force $P$ versus time $t$ and the pressure distribution $p$ on the $\mathrm{x}$-axis at $y=0$ and at the final time $T$ are given in Figs. 3(a) and 3(b) respectively. The same
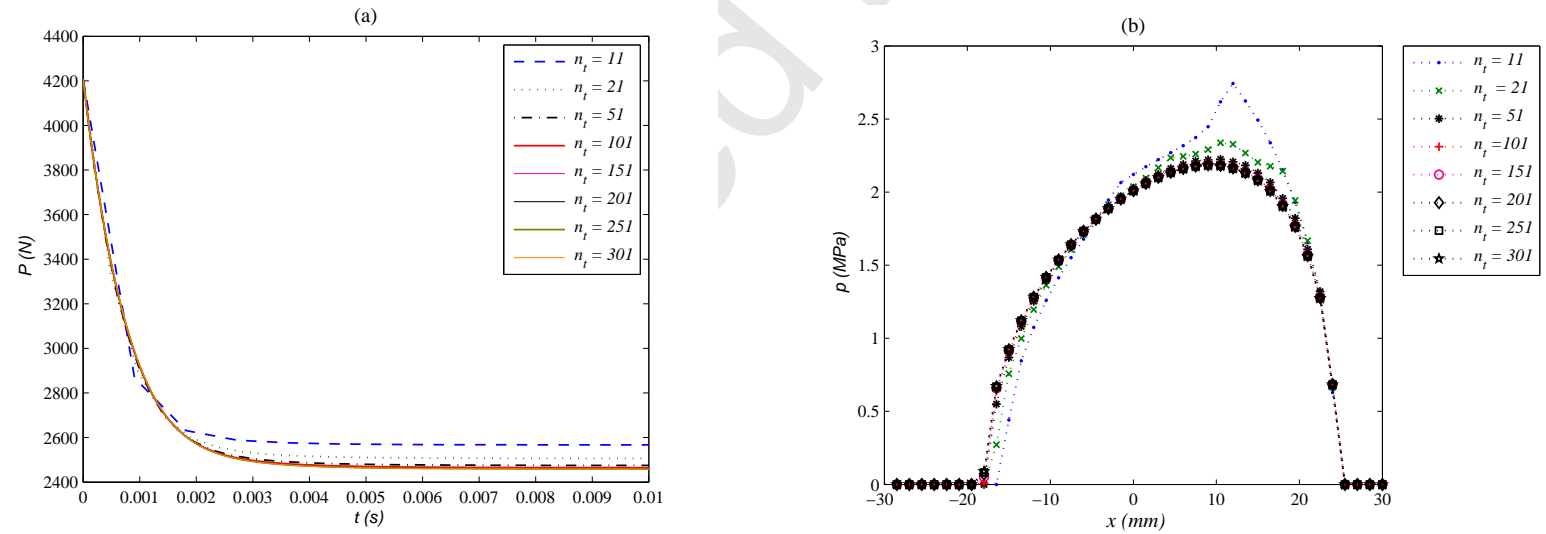

Fig. 3: Total force $P$ vs time and normal pressure $p$ on the $\mathrm{x}$-axis at $t=T$ for different time discretizations.

procedure was performed for $V_{x}=7.5 \mathrm{~m} / \mathrm{s}(27 \mathrm{~km} / \mathrm{h})$ and $30 \mathrm{~m} / \mathrm{s}(108 \mathrm{~km} / \mathrm{h})$. Convergence curves are given in Figs. 4(a) and 4(b) for the normalized total force $P / P_{\text {ref }}$ and the normalized contact pressure $p / p_{\text {ref }}$ at time $T$, where $P_{\text {ref }}$ and $p_{\text {ref }}$ are the values obtained for $n_{t}=301$. Note that the spatial resolution was $3.0 \mathrm{~mm}$ in these calculations. Figs. 4a and $4 \mathrm{~b}$ show a good convergence of the proposed algorithm in the time domain. The error of the calculation decreases steadily and rapidly with the time step.

\subsection{Comparison with the simple viscoelastic foundation model}

The new algorithm was compared with the simple viscoelastic foundation model used in Flom and Bueche (1959) for spheres, also described in Johnson (1985) for cylinders. A simple Voigt model (i.e. a single spring connected in parallel with a dashpot) is used in Flom and Bueche (1959) while the standard linear solid described in Eq. (19) is used by Johnson (1985). We propose here to derive the results in quasi-static conditions for a rigid sphere rolling on a visco-elastic fundation with a standard linear solid behavior. 

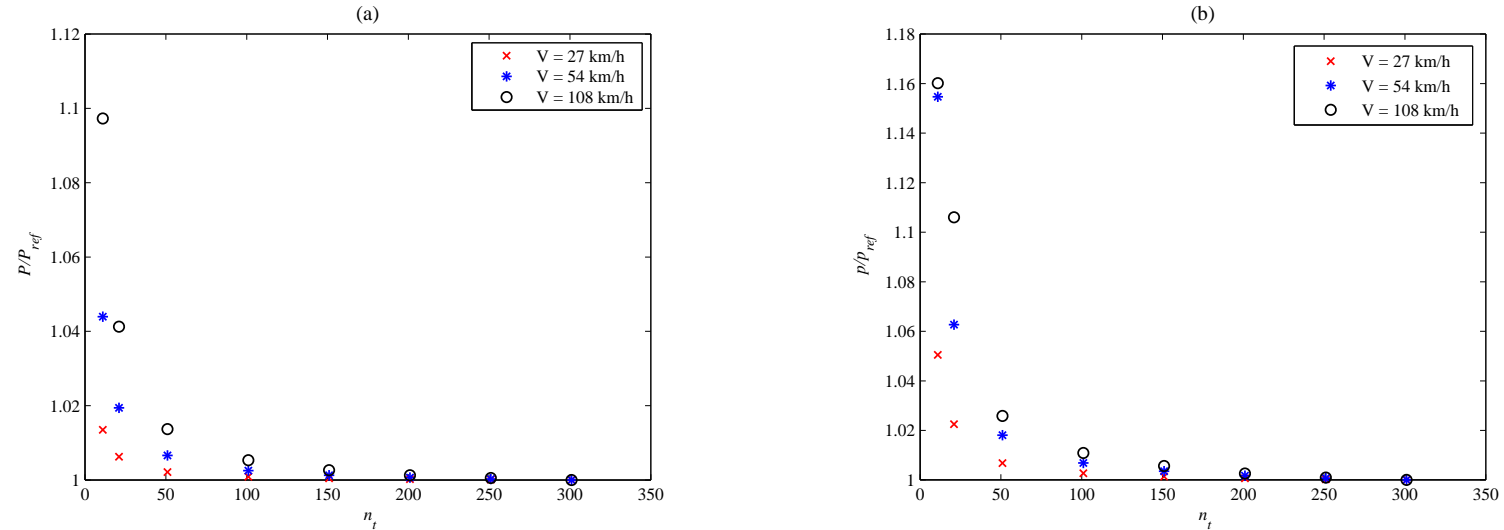

Fig. 4: Convergence of the method at different speeds for the total force $P$ and the contact pressure $p$ at time $T$.

In quasi-static conditions we have $x=-V t$ and $\partial z / \partial t=-V \partial z / \partial x$. Then the pressure distribution is linked to the surface geometry by:

$$
p(x, y)=-\frac{1}{h} \int_{\sqrt{a^{2}-y^{2}}}^{x} G\left(x-x^{\prime}\right) \frac{\partial z\left(x^{\prime}, y\right)}{\partial x^{\prime}} d x^{\prime}
$$

where $h$ is the depth of the viscoelastic foundation and $a$ is the radius of the contact area in static conditions. We assume that the sphere can be approximated by a paraboloid of revolution within the contact area (i.e. $x^{2} / R^{2}+y^{2} / R^{2} \ll 1$ and $a^{2} / R^{2} \ll 1$ ), so that:

$$
z(x, y)=\frac{1}{2 R}\left(a^{2}-x^{2}-y^{2}\right)
$$

Then integrating Eq. (20) gives a result similar to the cylinder case given by Johnson (1985):

$$
p(x, y)=\frac{K a^{2}}{R h}\left[\frac{1}{2}\left(1-x^{2} / a^{2}-y^{2} / a^{2}\right)+\beta \zeta(x / a-\alpha)+\beta \zeta(\alpha+\zeta)\left(1-e^{\frac{x / a-\alpha}{\zeta}}\right)\right]
$$

with $K=E_{\infty}, \alpha=\sqrt{1-y^{2} / a^{2}}, \beta=\left(E_{0}-E_{\infty}\right) / E_{\infty}$ and $\zeta=V \tau_{c} / a$.

The pressure distribution obtained with the new method proposed in the article was compared with the result of Eq. (22). A rigid sphere of radius $R=150 \mathrm{~mm}$ rolling at a constant speed $V_{x}=15 \mathrm{~m} / \mathrm{s}$ with a fixed penetration $\delta=4.2 \mathrm{~mm}$ was tested. The spatial resolution was $d x=d y=1.5 \mathrm{~mm}$. The number of time segments $n_{t}$ was 101 . The depth $h$ of the elastic foundation was calculated by identification of the total normal load with Hertz's theory following Johnson (1985), which gives:

$$
h=\frac{3 \pi\left(1-\nu^{2}\right) a}{16}
$$

with $\nu=0.5$ is the Poisson's coefficient of the half-space.

The normalized pressure distribution $p / p m$ is given at time $t=0$ and $t=\mathrm{T}=0.01 \mathrm{~s}$ in Fig. 5. In both cases (half-space or fundation model), it is clearly observed that the pressure distribution becomes asymmetrical for a rolling sphere and that the contact area at the final instant $T$ is no longer circular. This is due to the viscoelasticity of the material which relaxes more slowly than it is compressed. The same effect is illustrated in Fig. 6 for the pressure distribution on the $\mathrm{x}$-axis for $y=0$. While the contact areas in Fig. 5 are in the same order 

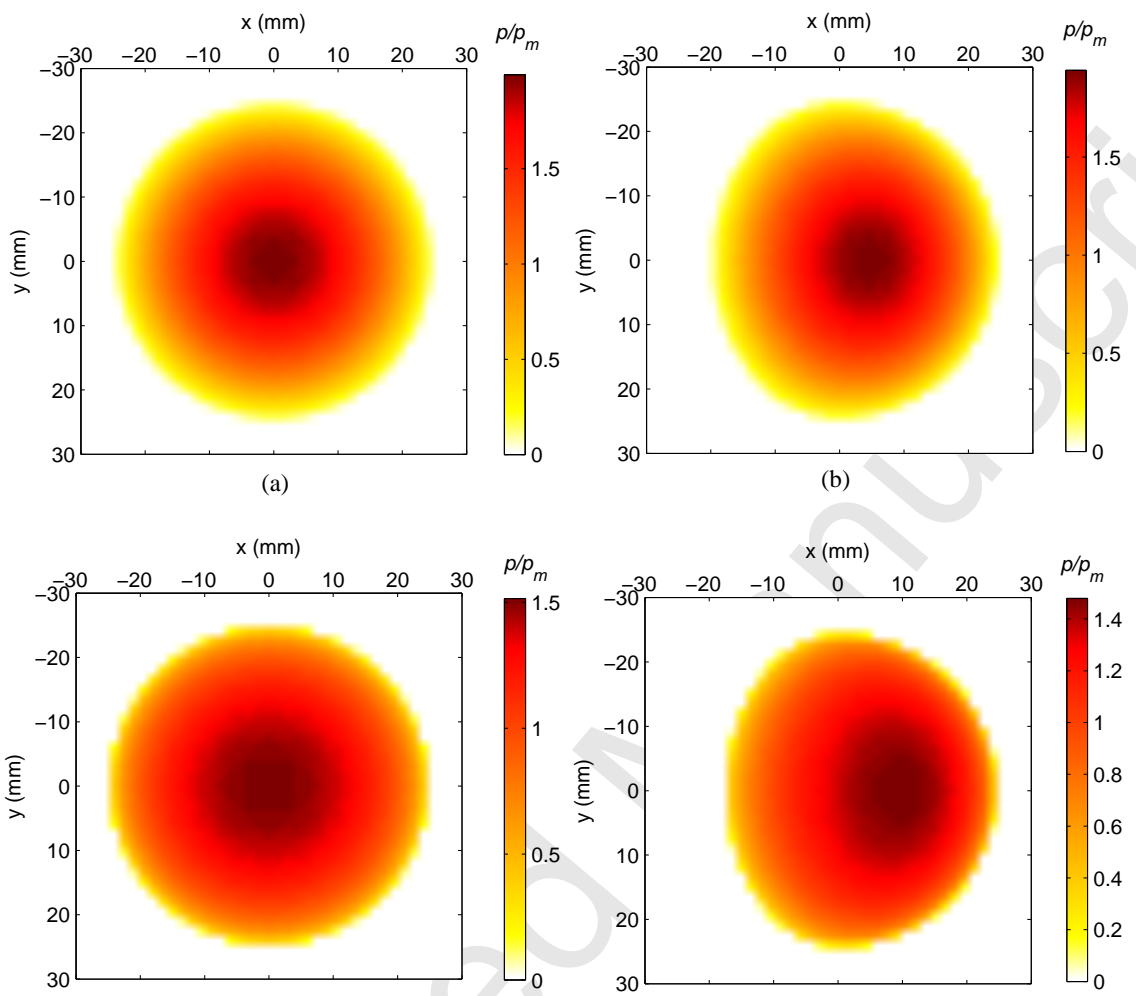

(c)

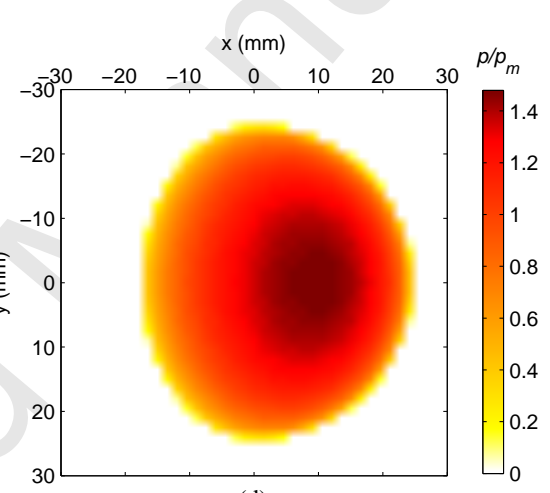

(d)

Fig. 5: Contact patches for the spherical indenter rolling on a viscoelastic foundation: (a) $V=0 \mathrm{~m} / \mathrm{s},(\mathrm{b}) V=$ $15 \mathrm{~m} / \mathrm{s}$ and on a viscoelastic half-space: (c) at time $t=0,(\mathrm{~d})$ at time $t=T$.

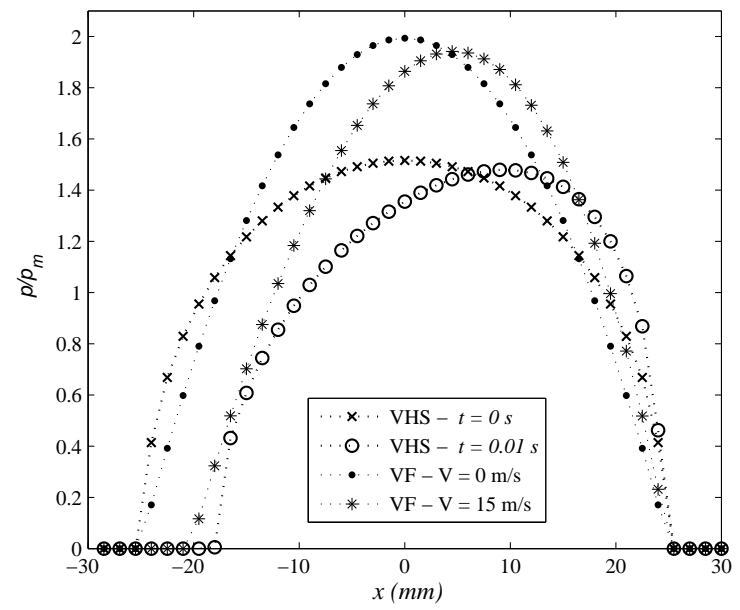

Fig. 6: Contact pressure distribution on the $x$-axis for $y=0$ for the spherical indenter at time $t=0$ and $t=T$. VHS: viscoelastic half-space, VF: viscoelastic foundation. 
and quite similar in shape, the pressure distribution obtained between the half-space and the fundation model are quite different. This may be due to the fact that interaction between contact elements is not taken into account in the foundation model. This drawback of the foundation model is underlined in the book of Johnson (1985) when comparing the results with those of Hunter (1961) in the two-dimensional case. Then the results of our model based on elasticity theory give more accurate results than the simple fundation model. This can be justify by the maximal value of the normalized pressure distribution $p / p_{m}$ in Fig. 6 which is closed to Hertz's theory for the half-space $\left(p_{\max } / p_{m} \simeq 1.5\right)$ while it is higher for the viscoelastic foundation $\left(p_{\max } / p_{m} \simeq 2.0\right)$.

\subsection{Influence of the indenter geometry}

The efficiency of the method was tested for indenters of different 3-D shapes. The rigid sphere was tested with the same parameters as in section 4.2. Then two cylinders of radius $R$ $=150 \mathrm{~mm}$, but with different width $l$ were tested at a constant speed of $V_{x}=15 \mathrm{~m} / \mathrm{s}$. The number of time segments $n_{t}$ was 101 . The first one was a short cylinder of width $l=R / 5=$ $30 \mathrm{~mm}$. The spatial resolution was $d x=d y=1.5 \mathrm{~mm}$ and the penetration $\delta=4.0 \mathrm{~mm}$. The second cylinder has a width $l=R=150 \mathrm{~mm}$ with a spatial resolution $d x=1.5 \mathrm{~mm}$ and $d y=$ $3.0 \mathrm{~mm}$ and a penetration $\delta=2.0 \mathrm{~mm}$. The pressure distributions for the two cylinders at time $T$ are given in Figs. 7.
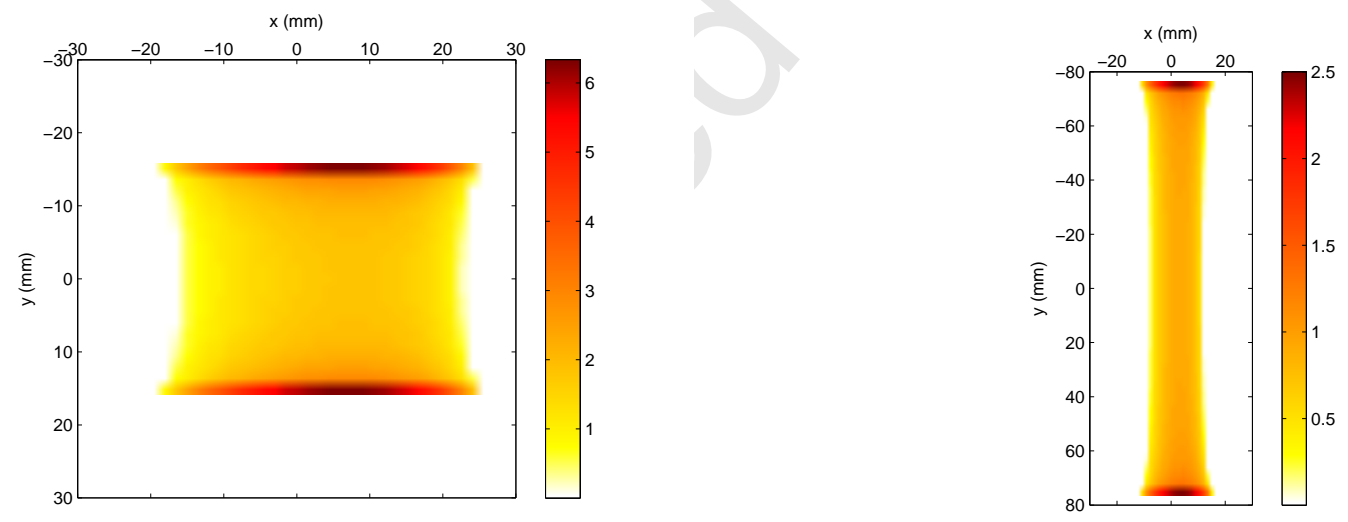

Fig. 7: Final contact patch for the short $(l=30 \mathrm{~mm})$ and the large $(l=150 \mathrm{~mm})$ cylinders.

As the sphere, the pressure distribution is still asymmetrical for the cylinders due to the viscoelastic behavior of the material. High pressure values are found at the top and bottom sides of the cylinders due to sharp edges, which can not be observed with two dimensional models. The normalized pressure distributions versus $x / a$, where $a$ is the radius of the contact area, are given in Fig. 8 for the three tested shapes. The results are quite similar (the dissymmetry is found for the three indenters), but differences are found in the amplitudes of $p / p_{m}$ due to the geometries of the indenters. This kind of result could find an application for studying the rolling resistance of pneumatic tyres on a road surface.

\subsection{Results for a varying speed}

Finally the method was tested for the spherical indenter with a speed $V_{x}$ sinusoidally varying with time:

$$
V_{x}(t)=V_{x}(1+\sin (2 n \pi t / T))
$$




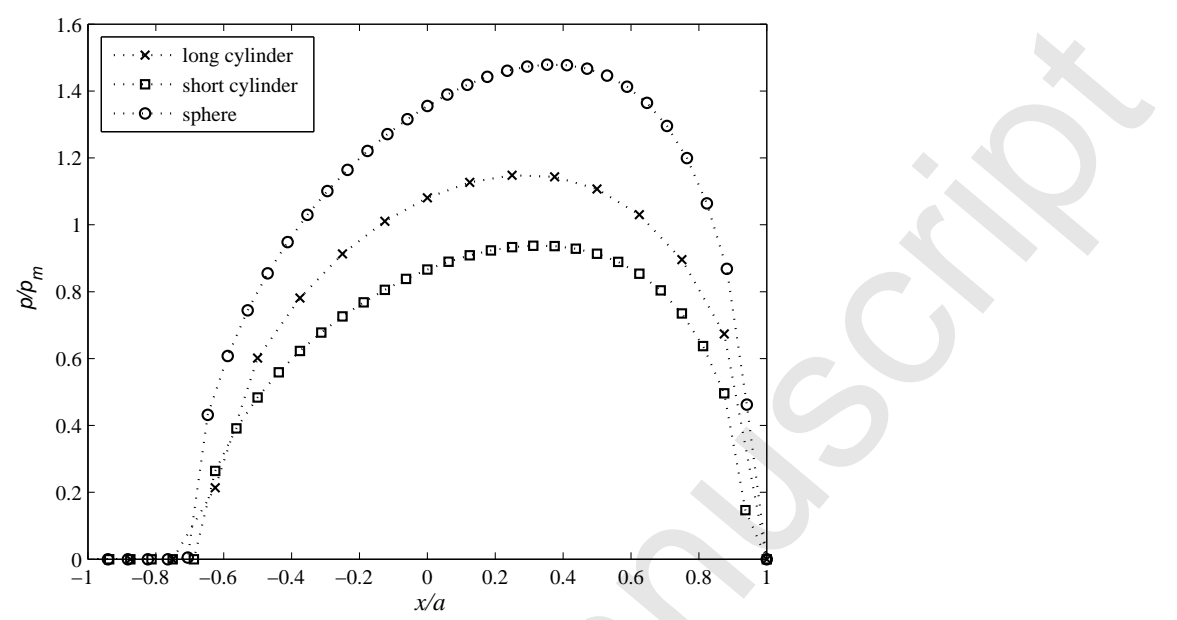

Fig. 8: Comparison of the pressure distribution on the $x$-axis for $y=0$ for the three tested geometries.

where $\mathrm{n}$ is the number of periods and $V_{x}=15 \mathrm{~m} / \mathrm{s}$. Results are presented in Figs. 9 for $n=4$. After a few periods, the total force and the pressure become periodic. The total force is delayed in comparison with the rolling speed. Within each period, the increasing part and the decreasing part of the force are slightly dissymmetric and the dissymmetry is obvious for the pressure history. In fact, the pressure increases more rapidly than it decreases. This dissymmetry, as that of pressure distribution, is due to the viscoelastic hysteresis. The same phenomenon was observed in the paper of Chertok et al. (2001) where the transient rolling of a rigid cylinder is studied on a two-dimensional semi-infinite body.

\section{Conclusions}

In this paper a new formulation was proposed for solving the time dependent rolling contact problem of a rigid body on a viscoelastic half-space in three dimensions. The viscoelastic problem was transformed into a succession of elastic like problems. Thus the transient rolling problem can be solved using a classical direct method scheme.

The results for a spherical indenter show a good convergence in time when the time step decreases. The dissymmetry of the contact patch is clearly observed when stationary rolling conditions are raised at a constant speed. This is in accordance with the previous results of the literature in two dimensions. The dissymmetry of the pressure distribution was also observed for a short and a long cylinder. Finally the method was tested for a rolling speed varying sinusoidally. The total force and the pressure distributions are delayed in comparison with the speed due to the viscoelastic hysteresis.

Future work will concern the rolling of a car tyre on a real road surface for application to rolling noise and rolling resistance. The method could also be used for studying the hysteretic friction of viscoelastic rubber sliding on three dimensional road surfaces. The main difficulty is due to the large number of asperities in contact with the tyre. The numerical method proposed in this article transforms the viscoelastic contact problem into a succession of elastic-like problems. Thus it could be combined with the two-scale approach developed in Cesbron et al. $(2008,2009)$ for tyre/road contact applications. 

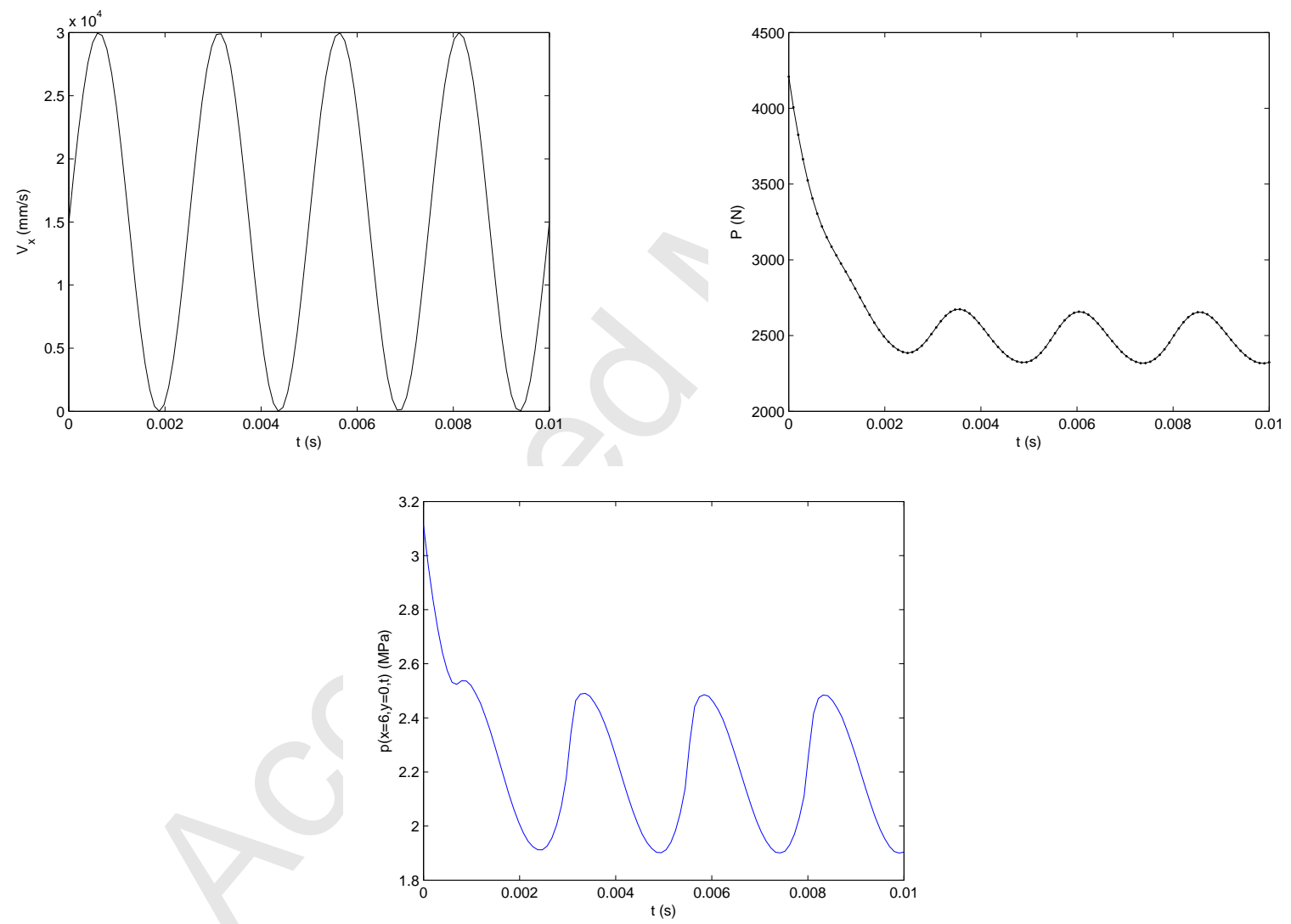

Fig. 9: Contact results for a sinusoidal speed (4 periods) for a spherical indenter. 


\section{References}

\section{References}

Brinkmeier, M., Nackenhorst, U., Petersen, S., von Estorff, O., 2008. A finite element approach for the simulation of tire rolling noise, Journal of Sound and Vibration 309 (1-2), 20 - 39.

Cesbron, J., Anfosso-Lédée, F., Yin, H. P., Duhamel, D., Le Houédec, D., 2008. Influence of road texture on tyre/road contact in static conditions, Road Materials and Pavement Design $9(4), 689-710$.

Cesbron, J., Yin, H. P., Anfosso-Lédée, F., Duhamel, D., Le Houédec, D., Feng, Z., 2009. Numerical and experimental study of multi-contact on an elastic half-space, International Journal of Mechanical Sciences 51 (1), 33-40.

Chan, S.H., Tuba, I.S., 1971. A finite element method for contact problems of solid bodies. International Journal in Mechanical Sciences 13, 615 - 639.

Chertok, D.L., Golden, J.M., Graham, G.A.C., 2001. Hysteretic friction for the transient rolling contact problem of linear viscoelasticity. Journal of Applied Mechanics - Transaction - ASME $68(4), 589-595$.

Flom, D.G., Bueche, A.M., 1959. Theory of rolling friction for spheres, Journal of Applied Physics 30(11), 1725-1730.

González, J. A., Abascal, R., 2006. Efficient stress evaluation of stationary viscoelastic rolling contact problems using the boundary element method: Application to viscoelastic coatings, Engineering Analysis with Boundary Elements 30 (6), 426 - 434.

Hunter, S., 1961. The rolling contact of a rigid cylinder with a viscoelastic half-space, ASME J. Appl. Mech 28, 611-617.

Johnson, K. L., 1985. Contact mechanics, Cambridge University Press.

Kalker, J. J., 1990. Three-dimensional elastic bodies in rolling contact, Vol. 2 of Solid mechanics and its applications, Kluwer Academic Publishers, Dordrecht, The Netherlands.

Kalker, J. J., 1991. Viscoelastic multilayered cylinders rolling with dry friction, Journal of Applied Mechanics - Transactions of the ASME 58(3), 666 - 679.

Kozhevnikov, I. F., Cesbron, J., Duhamel, D., Yin, H. P., Anfosso-Lédée, F., 2008. A new algorithm for computing the indentation of a rigid body of arbitrary shape on a viscoelastic half-space, International Journal of Mechanical Sciences 50 (7), 1194-1202.

Love, A. E. H., 1952. A treatise on the mathematical theory of elasticity, Cambridge University Press.

Morland, L., 1962. A plane problem of rolling contact in linear viscoelasticity theory, ASME J. Appl. Mech. 29, 345-352. 
Nackenhorst, U., 2004. The ALE-formulation of bodies in rolling contact: Theoretical foundations and finite element approach, Computer Methods in Applied Mechanics and Engineering 193 (39-41), 4299 - 4322.

Oden, J., Lin, T., 1986. On the general rolling contact problem for finite deformations of a viscoelastic cylinder, Computer Methods in Applied Mechanics and Engineering 57 (3), 297 367.

Padovan, J., Paramadilok, O., 1985. Transient and steady state viscoelastic rolling contact, Computers \& Structures 20 (1-3), 545 - 553.

Tabor, D., 1952. The mechanism of rolling friction, Philos. Mag. 43, 1055-1059.

Wang, G., Knothe, K., 1993. Stress analysis for rolling contact between two viscoelastic cylinders, Journal of Applied Mechanics - Transactions of the ASME 60(2), 310 - 317. 